\title{
Comparisons of the Clinical and Radiological Features and Surgical Management of Posterior Fossa Meningiomas and Acoustic Neuromas
}

\author{
C.H. Tator, E.G. Duncan and D. Charles
}

\begin{abstract}
Next to acoustic neuromas, meningiomas are the most common benign tumour of the posterior fossa. This paper reviews the personal experience of one of the authors with the management of 26 posterior fossa meningiomas and 212 acoustic neuromas. The aim was to compare the clinical and radiological features of these two types of posterior fossa tumours to determine whether they can be differentiated preoperatively. The study also aimed at analysing the surgical treatment of posterior fossa meningiomas. The results showed that posterior fossa meningiomas can usually be differentiated from acoustic neuromas on the basis of clinical and radiological features. Preoperative differentiation aids surgical management, especially for selection of surgical approach. In most instances, posterior fossa meningiomas can be totally resected with minimal morbidity and mortality.

RÉSUMÉ: Comparaisons entre les manifestations cliniques et radiologiques et le traitement chirurgical des méningiomes de la fosse postérieure et des neurinomes acoustiques Après le neurinome acoustique, le méningiome est la tumeur bénigne la plus fréquente de la fosse postérieure. Cet article rapporte l'expérience personnelle de l'un des auteurs dans le traitement de 26 méningiomes de la fosse postérieure et de 212 neurinomes acoustiques. Le but de ce travail était de comparer les manifestations cliniques et radiologiques de ces deux types de tumeurs de la fosse postérieure afin de déterminer si l'on peut les différencier avant la chirurgie. Nous voulions également analyser le traitement chirurgical des méningiomes de la fosse postérieure. Les résultats ont montré que les méningiomes de la fosse postérieure peuvent habituellement être différenciés par leurs manifestations cliniques et radiologiques. Un diagnostic pré-opératoire précis aide à déterminer la conduite chirurgicale, surtout en ce qui a traite à l'approche chirurgicale. Dans la plupart des cas, les méningiomes de la fosse postérieure peuvent être réséqués complètement avec un taux de morbidité et de mortalité minime.
\end{abstract}

Can. J. Neurol. Sci. 1990; 17:170-176

Next to acoustic neuromas, meningiomas are the most frequent benign tumour in adults in the posterior cranial fossa, including the cerebellopontine angle (CPA).' These two types of tumours can produce similar clinical and radiographic features. In order to improve the precision of diagnosis, the records of 270 consecutive cases of primary posterior fossa mass lesions were examined to document the features which assist in distinguishing between meningiomas and acoustic neuromas. This series contained 212 acoustic neuromas and 26 meningiomas. It is important to establish the diagnosis preoperatively to improve the results of surgery, especially by assisting in the selection of the most appropriate operative approach. Although most posterior fossa meningiomas should be removed through a suboccipital approach, some should be approached through translabyrinthine, transcochlear, subtemporal or combined approaches, especially those which have infiltrated the tentorium and have supratentorial extension, or which have grown anteriorly through the tentorial hiatus. Preoperative diagnosis of meningiomas is also important for delineation of the arterial supply to assist surgical dissection and selection of patients for preoperative embolization.

\section{Clinical Material and Methods}

This report is based on 270 consecutive patients with primary tumours of the posterior fossa managed by one of the authors (C.H.T.) between 1970 and 1985. The patients were treated at either Sunnybrook Medical Centre or the Toronto Western Hospital, University of Toronto. The follow-up was obtained

From the Division of Neurosurgery and Department of Otolaryngology, Toronto Hospital, Toronto Western Division, University of Toronto, Toronto

Reprint requests to: C.H. Tator, M.D., Toronto Western Hospital, University of Toronto, 399 Bathurst Street, Suite 2-003, E.C.W., Toronto, Ontario, Canada M5T 2S8 
Table 1: 270 Primary Tumours of the Posterior Fossa

\begin{tabular}{lrr}
\hline \hline & Number & Percent \\
\hline Acoustic Neuroma & 212 & 78.5 \\
Meningioma & 26 & 9.6 \\
Glioma & 12 & 4.4 \\
— Cerebellar Astrocytoma (7) & & \\
— Brainstem Astrocytoma (3) & & \\
- Cerebellar Glioblastoma (2) & 4 & 1.5 \\
Facial Neuroma & 3 & 1.1 \\
Arachnoid Cyst & 3 & 1.1 \\
Epidermoid Cyst & 3 & 1.1 \\
Medulloblastoma & 2 & 0.7 \\
Haemangioendothelioma & 1 & 0.4 \\
Glomus Jugulare & 1 & 0.4 \\
Giant Cell Tumour & 1 & 0.4 \\
Ependymoma & 1 & 0.4 \\
Haemangiocytoma & 1 & 0.4 \\
Dysplastic Gangliocytoma & 270 & 100.0 \\
\hline
\end{tabular}

Table 2: Disability at Presentation

\begin{tabular}{lcccc}
\hline \hline & \multicolumn{2}{c}{ Acoustic Neuroma } & \multicolumn{2}{c}{ Meningioma } \\
Disability* & Number & Percent & Number & Percent \\
\hline Mild & 189 & 89.2 & 20 & 76.9 \\
Moderate & 17 & 8.0 & 4 & 15.4 \\
Severe & 6 & 2.8 & 2 & 7.7 \\
\hline TOTAL & 212 & 100.0 & 26 & 100.0 \\
\hline
\end{tabular}

*See text for description.

\begin{tabular}{lcccc}
\hline Table 3: Location of Tumours & & & \\
\hline \hline Location of & Acoustic & Neuroma & \multicolumn{2}{c}{ Meningioma } \\
Tumours & Number & Percent & Number & Percent \\
\hline Intracanalicular & 4 & 1.9 & 0 & 0.0 \\
CPA & 208 & 98.1 & 0 & 0.0 \\
Tentorium & 0 & 0.0 & 12 & 46.2 \\
Petrous Bone & 0 & 0.0 & 12 & 46.2 \\
Foramen Magnum & 0 & 0.0 & 1 & 3.8 \\
Torcula & 0 & 0.0 & 1 & 3.8 \\
\hline TOTAL & 212 & 100.0 & 26 & 100.0 \\
Bilateral & 7 & 3.3 & 2 & 7.7 \\
Neurofibromatosis & 7 & 3.3 & 2 & 7.7 \\
\hline
\end{tabular}

\section{Table 4: Size of Tumours}

\begin{tabular}{lcccc}
\hline & \multicolumn{2}{c}{ Acoustic Neuroma } & \multicolumn{2}{c}{ Meningioma } \\
Size of Tumours & Number & Percent & Number & Percent \\
\hline Small $[<20 \mathrm{~mm}]$ & 76 & 35.9 & 6 & 23.1 \\
Medium $[20-40 \mathrm{~mm}]$ & 105 & 49.5 & 11 & 42.3 \\
Large $[>40 \mathrm{~mm}]$ & 31 & 14.6 & 9 & 34.6 \\
\hline TOTAL & 212 & 100.0 & 26 & 100.0 \\
Mean $(\mathrm{mm})$ & & & \multicolumn{2}{c}{35} \\
Median (mm) & 26 & & \multicolumn{2}{c}{35} \\
\end{tabular}

in all cases by clinical examination and in recent cases by computerized tomography (CT) and magnetic resonance (MR) imaging.

The selection of operative approach was made on the basis of the presumed diagnosis, the size and location of the tumour and the presence of useful preoperative hearing. If a meningioma was suspected in the CPA, a suboccipital approach was used if the tumour appeared confined to the posterior aspect of the petrous temporal bone. The translabyrinthine and combined lateral approaches were selected for those meningiomas with supratentorial growth, extension forwards through the tentorial hiatus, or invasion of the petrous temporal bone. Conversely, if an acoustic neuroma was suspected, the approach selected depended on the size of the tumour and the presence of serviceable hearing. Small tumours in patients with serviceable hearing were removed through a suboccipital approach, whereas large tumours in patients with poor hearing were removed through translabyrinthine or lateral approaches. The details of selection of approaches for acoustic neuromas have been described elsewhere. 2.5

If a meningioma was suspected angiograms were almost always obtained, but with most acoustic neuromas, angiograms were seldom obtained. In this series, none of the patients had preoperative embolization.

Table 1 shows the final diagnosis in the 270 cases. Acoustic neuromas comprised 212 cases $(78.5 \%)$ and the 26 meningiomas were the second most frequent type (9.6\%). There were 12 gliomas of various types and a variety of other less common tumours. Some of the clinical details of the 212 acoustic neuromas have already been reported. ${ }^{2}$

The records of these 270 cases were analyzed with the use of a microcomputer and the SAS for PC (TM) statistical software package. Differences between the acoustic neuroma and meningioma groups were determined by the Chi-square or t-statistic with the level of significance at $p<.05$.

\section{Results}

\section{Age}

The patients with meningiomas and acoustic neuromas were almost identical in age at the time of presentation, although with the meningiomas, the 31-40 age group was the most common age by decade, whereas the most common with the acoustic neuromas was the 41-50 age group. The average age of patients with meningiomas was 46.0 years and for acoustic neuromas 48.4 years, and these were not significantly different ( $p>05)$.

\section{Sex}

There was a greater preponderance of females in the meningioma group $(73.1 \%)$ as compared with the acoustic neuroma group (48.1) and this was a significant difference $(p<.05)$.

\section{Symptoms and Signs}

The presenting symptoms and signs were classified as mild, moderate or severe disability. Mild disability included hearing loss, tinnitus, mild headaches, episodic ataxia, facial numbness or partial facial weakness. In addition to the above, moderate disability also included nausea and vomiting, severe headaches, persistent mild ataxia, or complete facial palsy. Severe disability included the above, with the addition of dementia, incontinence, 
severe ataxia, inability to walk, or papilloedema. Table 2 shows that meningiomas tended to cause greater difficulty with $23.1 \%$ of patients having moderate or severe disability, whereas only $10.8 \%$ of the patients with acoustic neuroma had moderate or severe disability, although these differences were not significant ( $\mathrm{p}>$.05).

The presenting symptoms were related to the size and location (Table 3) of the tumours. All the petrous meningiomas and most of the tentorial meningiomas had hearing loss as a present-

Table 5: Radiological Diagnosis of Acoustic Neuroma vs. Meningioma in the Cerebellopontine Angle

\begin{tabular}{|c|c|c|}
\hline & Acoustic Neuroma & Meningioma \\
\hline \multicolumn{3}{|l|}{ Plain Films } \\
\hline $\begin{array}{l}\text { Internal Auditory } \\
\text { Canal }\end{array}$ & $\begin{array}{l}\text { Enlarged in } 90 \% \\
\text { or more }\end{array}$ & $\begin{array}{l}\text { Normal in } \\
\text { almost all }\end{array}$ \\
\hline Calcification & Never & Occasional \\
\hline Petrous Apex & Seldom Eroded & May be eroded \\
\hline $\begin{array}{l}\text { Hyperostosis, } \\
\text { Exostosis }\end{array}$ & Absent & May be present \\
\hline \multicolumn{3}{|l|}{ CT } \\
\hline Base of Tumour & $\begin{array}{l}\text { Smaller, with acute } \\
\text { angles }\end{array}$ & Larger, sessile \\
\hline Non-Enhanced CT & Mass may not be seen & Mass often seen \\
\hline Enhanced CT & Enhancing mass & Enhancing mass \\
\hline Cystic & Common & Less common \\
\hline \multicolumn{3}{|l|}{ Angiography } \\
\hline $\begin{array}{l}\text { Angiographic Blush } \\
\text { Large Feeding }\end{array}$ & Uncommon & Common \\
\hline Vessels & Uncommon & Common \\
\hline \multicolumn{3}{|l|}{$\mathbf{M R}$} \\
\hline Tumour & Always & Rare \\
\hline VII, VIII nerves & $\begin{array}{l}\text { Inseparable from } \\
\text { tumour }\end{array}$ & $\begin{array}{l}\text { Distinguishable } \\
\text { from tumour }\end{array}$ \\
\hline
\end{tabular}

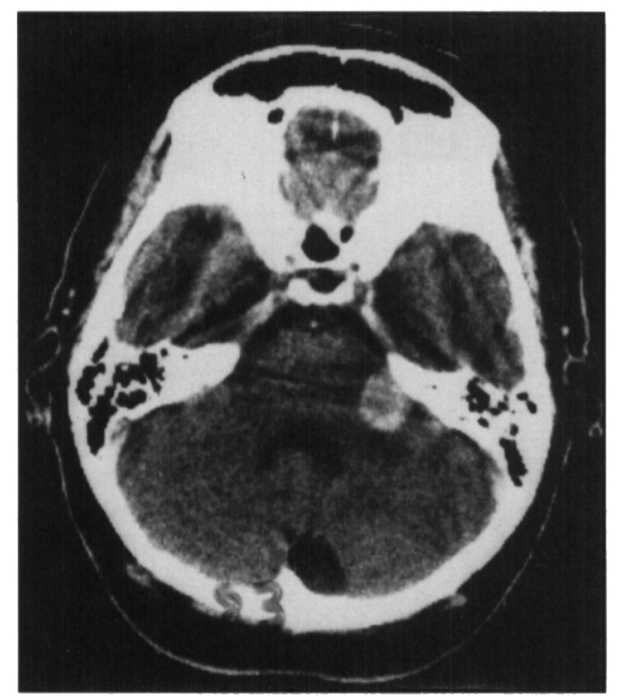

Figure I - Contrast enhanced CT scan of acoustic neuroma. Note the small base of the tumour along the posterior aspect of the petrous bone and the acute angle between the posterior aspect of the tumour and the temporal bone.
Table 6: Surgical Treatment

\begin{tabular}{lcccr}
\hline \hline Type of Procedure & \multicolumn{2}{c}{ Acoustic Neuroma } & \multicolumn{2}{c}{ Meningioma } \\
Number & Percent & Number & Percent \\
\hline Excision - Total & 162 & 76.4 & 17 & 65.4 \\
Excision - Partial & 8 & 3.8 & 4 & 15.4 \\
Shunt Only & 4 & 1.9 & 1 & 3.9 \\
Shunt + Total Excision & 14 & 6.6 & 2 & 7.7 \\
Non-Operative & 24 & 11.3 & 2 & 7.7 \\
\hline TOTAL & 212 & 100.0 & 26 & 100.0 \\
\hline
\end{tabular}

Table 7: Surgical Approach (Excluding Shunts)

\begin{tabular}{lcccc}
\hline \hline Type of Approach & \multicolumn{2}{c}{$\begin{array}{c}\text { Acoustic Neuroma } \\
\text { Number }\end{array}$} & $\begin{array}{c}\text { Mercent } \\
\text { Number }\end{array}$ & $\begin{array}{c}\text { Numatioma } \\
\text { Percent }\end{array}$ \\
\hline Suboccipital & 85 & 46.2 & 14 & 60.9 \\
Translabyrinthine & 37 & 20.1 & 1 & 4.3 \\
Expanded & & & & 34.8 \\
Translabyrinthine & 62 & 33.7 & 8 & 3 \\
\hline TOTAL & 184 & 100.0 & 23 & 100.0 \\
\hline
\end{tabular}

ing symptom, and thus, the initial diagnosis in many of these cases was acoustic neuroma. Facial numbness was a presenting symptom in 10 of the 24 cases of tentorial or petrous meningiomas, but was rarely a presenting symptom in the patients with acoustic neuroma.

\section{Location of the Tumours}

The principal location of the tumours is shown in Table 3 , although it is acknowledged that many of the meningiomas were attached to more than one structure. The most common location for the acoustic neuroma was the CPA, with only 4 tumours confined solely to the internal auditory canal. The most common principal site of attachment of the meningiomas was the petrous bone in 12 patients $(46.2 \%)$ and the tentorium in 12 patients (46.2\%). All 24 of these tentorial or petrous meningiomas were classified as CPA tumours because they comprised mass lesions in the angle. The two other meningiomas arose at the foramen magnum and the torcula in one case each.

In seven cases, the acoustic neuromas were bilateral, and in two cases, the meningiomas were bilateral. In all nine of these cases, the patients had neurofibromatosis. In one of the bilateral meningioma cases, the tumours were confined to the internal auditory canal. This case was classified as petrous temporal in location because the tumours arose from the dura in that location.

\section{Hydrocephalus}

Hydrocephalus was present in 30 cases of acoustic neuroma (14.2\%) and 6 cases of meningioma (23.1\%).

\section{Tumour Size}

The size of the tumours was classified into small (less than $20 \mathrm{~mm}$ ), medium $(20-40 \mathrm{~mm}$ ), and large (over $40 \mathrm{~mm}$ ), as shown in Table 4 . In the medium and large categories there were more meningiomas $(76.9 \%)$ than acoustic neuromas $(64.1 \%)$. The average size meningioma was $35 \mathrm{~mm}$, which was significantly larger than the average size acoustic neuroma, which was $26 \mathrm{~mm}(\mathrm{p}<.05)$. 


\section{Radiological Diagnosis}

When the meningiomas were located principally in the CPA, either arising from the petrous temporal bone or from the tentorium, they were often difficult to distinguish radiologically from acoustic neuromas. Table 5 shows the features on plain films, $\mathrm{CT}$, angiography and MR which were considered helpful in differentiating the two. On the plain films or conventional tomograms, the internal auditory canal was enlarged in almost all patients with acoustic neuromas, but was seldom enlarged by meningiomas. On the CT scan, the lateral edge of the acoustic neuroma adjacent to the posterior aspect of the petrous temporal bone was often small with acute angles, especially posteriorly (Figure 1) whereas the meningiomas tended to be sessile (Figures 2 and 3). MR is the best radiological method of differentiating the two types. The MR showed that the acoustic neuromas extended into the internal auditory canal, usually to the lateral limit of the canal, and that the tumour mass was indistinguishable from the seventh and eighth cranial nerves. In contrast, the MR showed that the meningiomas were mainly extracanalicular with only a small extension lateral to the porus, and that often the internal auditory canal was not expanded (Figure 4). Moreover, in high resolution MR studies, the seventh and eighth cranial nerves could be distinguished from the meningiomas and were identifiable in the internal auditory canal. An angiographic blush and large feeding vessels were usually present in meningiomas, but uncommon in acoustic neu-

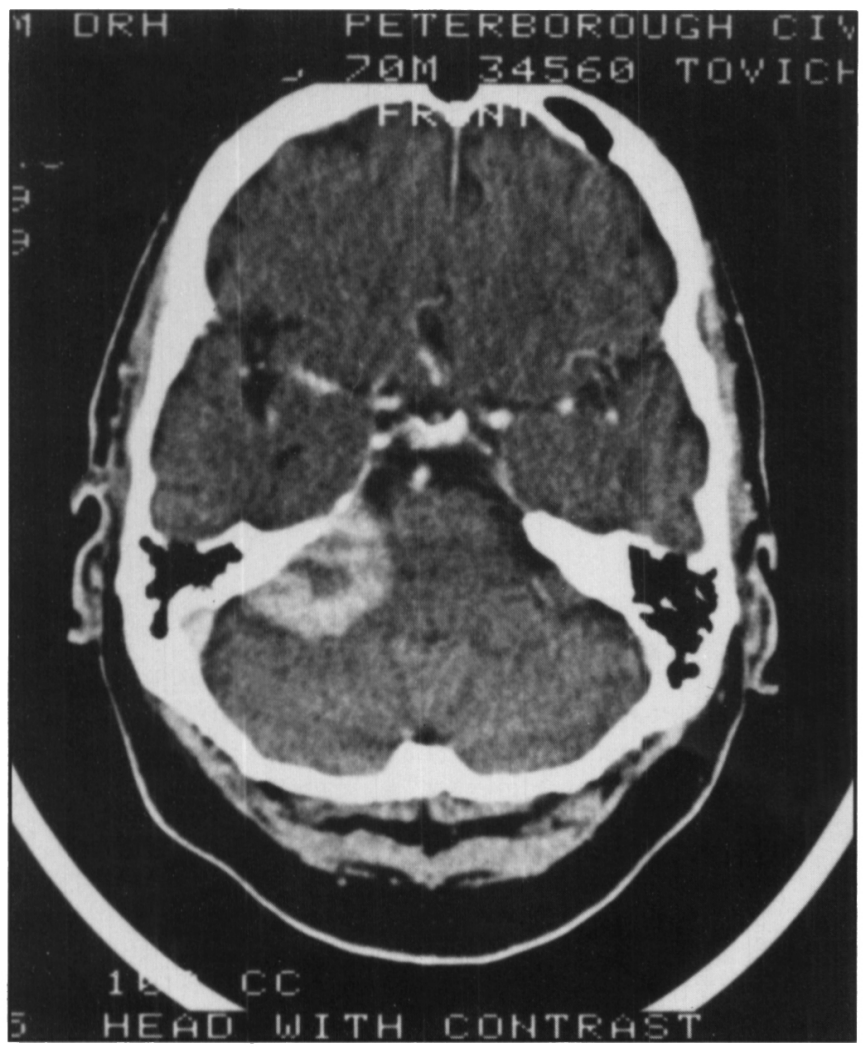

Figure $2-$ Contrast enhanced CT of perrous meningioma. Note the broad, sessile base of the tumour along the posterior margin of the petrous temporal bone. The tumour is also arising from the edge of the tentorium at the anteromedial aspect of the tumour. The low density area in the centre of the tumour represents a cyst.
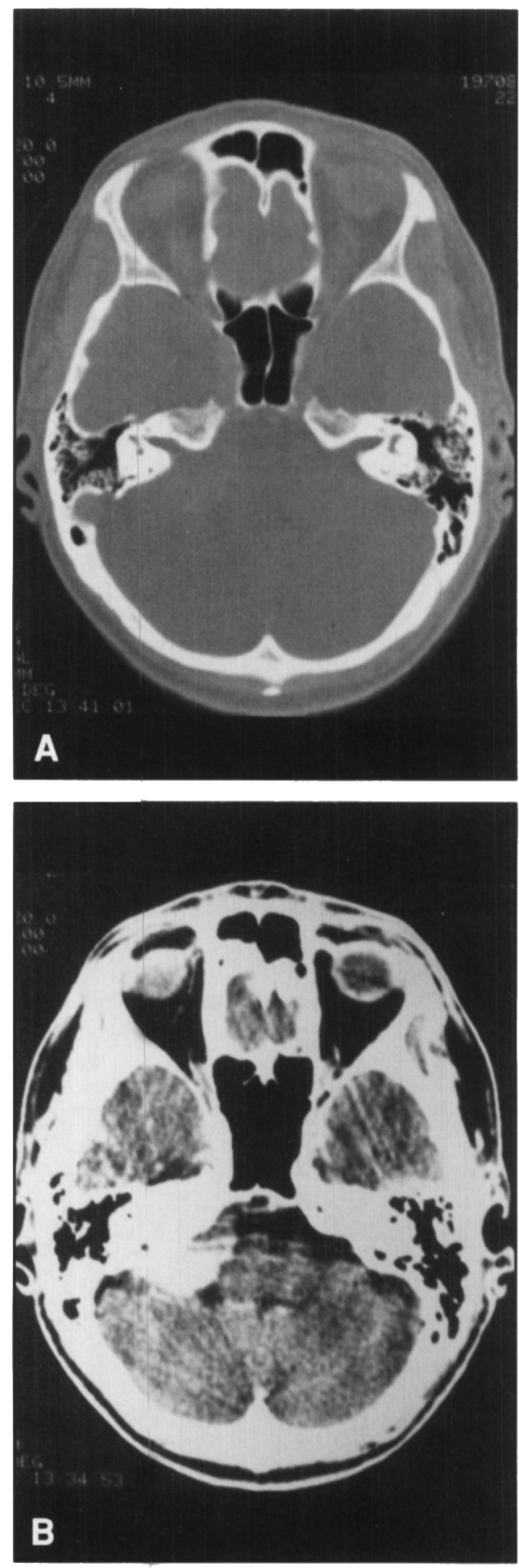

Figure $3-A$. Above. Bone window $C T$ of petrous meningioma showing normal porus acousticus and internal auditory canal. B. Below. Non-enhanced CT of the same patient as $A$ showing sessile, dense meningioma aris. ing adjacent to posterior aspect of petrous temporal bone. The low' density area of the tumour anteromedially is due to artifact. 
romas. Prior to MR, the precision of radiological differentiation of CPA meningiomas and acoustic neuromas was about $50 \%$, but with the addition of MR recent cases have been differentiated with almost $100 \%$ precision.

\section{Surgical Treatment}

As outlined in the previous report of the 212 cases of acoustic neuromas, ${ }^{2}$ the tumours were removed surgically in 184 patients and in an additional 4 patients, only a shunt operation was performed (Table 6). The remaining 24 patients with acoustic neuroma were not operated upon, mainly elderly patients with small tumours. Of the 26 patients with meningioma, 21 underwent total or partial excision of the tumours, one was treated with a shunt only, two had shunts and total excision of the tumours and two were not operated upon, one an elderly patient with a small tumour and the other an elderly patient with an extensive tumour at the torcula.

The considerations involved in the selection of the operative approach to CPA meningiomas arising from the petrous temporal bone or tentorium were somewhat different to those for acoustic neuromas which were detailed in our other publications. ${ }^{2-5}$ As shown in Table 7, most CPA meningiomas were operated on through a suboccipital approach $(60.9 \%)$, especially when hearing was serviceable, defined as a speech reception threshold less than 50 decibels and speech discrimination score more than $60 \%$. The expanded translabyrinthine approach was selected for large tumours with an anterior extension through the tentorial hiatus or upwards through the tentorium into the middle fossa. In the latter cases, the expanded translabyrinthine approach and the addition of a temporal craniectomy or bone flap and division of the tentorium were especially helpful for removing the middle fossa component.

At surgery, the acoustic neuromas generally had a smooth surface and were well encapsulated by a thin tumour capsule

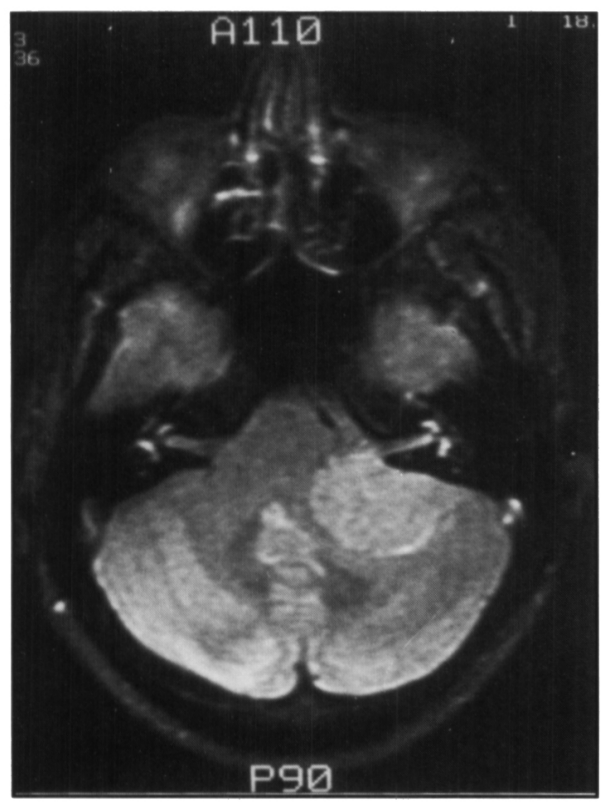

Figure 4-MR of petrous meningioma. Note that the tumour does not extend into the internal auditory canal, and that the canal is not expanded. and arachnoid, whereas the meningiomas were usually not covered by arachnoid and had the characteristic "pebbly" surface. With meningiomas, the seventh and eighth cranial nerves were generally found draped over the posterior or lateral aspects of the tumours, whereas with acoustic neuromas these nerves were almost always displaced anteriorly and adherent to the anteromedial aspect of the tumours. With the larger acoustic neuromas, large blood vessels including the posterior and anterior inferior cerebellar arteries and the superior cerebellar arteries were almost always stretched over the tumour capsule, whereas in several of the meningiomas, these vessels were incorporated into the tumours or engulfed by them.

\section{Results}

Of the 23 cases in which the meningiomas were approached surgically, six patients had a complete facial paralysis postoperatively which did not resolve in time, and all were treated with facial-hypoglossal anastomosis. There was a mild facial palsy postoperatively in five patients, all of which resolved with little or no cosmetic deficit, and no facial palsy occurred in the remaining 12 patients. Hydrocephalus occurred postoperatively in two patients, and both required shunts. Two patients had CSF rhinorrhea postoperatively, both after translabyrinthine approaches, one of which developed meningitis which responded to antibiotic therapy without residual deficit. One patient had an intracerebellar hemorrhage postoperatively which required evacuation, and one patient had a brainstem and cerebellar infarct due to clipping of the superior cerebellar artery, necessitated by uncontrollable haemorrhage from this vessel which was encountered within the substance of the tumour. Although this patient was left with severe ataxia and dysarthria, he is ambulatory with one cane. There were two deaths in the acoustic neuroma group, but none in the meningioma group. Indeed, all patients with meningioma except the patient described above with the infarct in the distribution of the superior cerebellar artery made an excellent recovery and were fully ambulatory.

\section{Discussion}

Castellano and Ruggiero' and Miller6 classified posterior fossa meningiomas on the basis of their dural attachment and identified six types: petrous ridge, tentorial, foramen magnum, clivus, cerebellar convexity, and tela choroidea of the fourth ventricle. The present series contained examples of only the first three types and added another location, the torcula. Many of the CPA tumours in the present series which arose from either the petrous bone or from the tentorium also spread to the clivus, but there were none which were primarily clival.

In 1984, Sekhar and Jannetta ${ }^{7}$ reviewed the eight previously reported series of cases of CPA meningiomas and emphasized the markedly reduced mortality rate achieved in the more recent series. The older series of cases $1,8,9,10,11,12$ had mortality rates ranging up to $45 \%$ for total excision and as high as $60 \%$ for partial excision. In contrast, the three recent series of cases including that of Sekhar and Jannetta had no deaths in either the total or partial resection groups, 7,13,14 and the incidence of total resection was much higher. The present series with no fatalities and a high incidence of total resection is in keeping with the recent results achieved elsewhere. These improvements in results are attributable to many factors including the use of the 
operating microscope for identification of the large vessels and nerves, and intraoperative monitoring of brainstem and cranial nerve function. ${ }^{2}$ In addition, the laser has been very useful for firm, vascular meningiomas.

Accurate preoperative diagnosis of the pathological nature of a CPA tumour also underlies the improved operative results. As shown in Table 1, the acoustic neuroma is by far the most common type of primary posterior fossa tumour, and the meningiomas are the next comprising approximately $10 \%$. In addition to the lesions shown in Table 1, the CPA may contain secondary tumours and other lesions, such as aneurysms of the vertebral and basilar arteries and their branches. In general, the meningioma is most frequently mistaken for an acoustic neuroma preoperatively because the signs, symptoms and radiological features may be quite similar. Sekhar and Jannetta ${ }^{7}$ pointed out that both meningiomas and acoustic neuromas caused hearing impairment although the latter more frequently, and that meningiomas produced less labyrinthine dysfunction, but more trigeminal neuralgia and facial numbness. The findings in the present series were similar. Indeed, two patients with meningiomas in the present series had severe trigeminal neuralgia. In the present series, the ages of the patients with acoustic neuromas and meningiomas were almost identical. The meningiomas showed a female to male ratio of approximately $3: 1$; whereas acoustic neuromas occurred almost equally in males and females, and this difference has been noted by others.

In most cases, acoustic neuromas and CPA meningiomas can now be distinguished by their radiological features. Of particular importance is the almost constant finding of enlargement of the internal auditory canal with acoustic neuromas, a finding seldom present with meningiomas (Figure 3). Meningiomas may erode the petrous apex, a finding seldom present with acoustic neuromas. Although hyperostosis and exostosis are not common, the presence of these findings would indicate a meningioma. Acoustic neuromas tend to be rounder, with a smaller base against the posterior aspect of the petrous temporal bone and acute angles, whereas the meningiomas more frequently appeared to be sessile (Figures 1-3). Acoustic neuromas may not be detected on an unenhanced $\mathrm{CT}$, whereas meningiomas are usually seen on an unenhanced CT. In our series, meningiomas, tended to be larger than acoustic neuromas (Table 4).

MR is probably the most useful test for differentiating the two types of tumours. Acoustic neuromas can be seen incorporating the seventh and eighth cranial nerves and growing from the CPA into an expanded internal auditory canal, whereas meningiomas usually protrude only slightly through the porus into the canal. Moreover, the cranial nerves can often be identified in the canal distinct from the tumour. With meningiomas, angiography may show a characteristic blush and large feeding vessels. However, as shown by Theron and Lasjaunias, ${ }^{15}$ some acoustic neuromas may show similar angiographic findings.

For three reasons, it is important to differentiate acoustic neuromas and meningiomas preoperatively. Firstly, a preoperative angiogram should be performed in patients with meningiomas to identify the exact location of the major vessels. In addition, well vascularized meningiomas can be embolized if there are large feeding vessels from the external carotid circulation, such as the ascending pharyngeal artery. Angiograms are usually not indicated in patients with acoustic neuromas. The second major reason for differentiating the tumours preoperatively is to aid in the selection of the operative approach. With acoustic neuromas, especially if the tumours are moderately large and have already produced an irreparable hearing loss, the translabyrinthine approach is preferred by some surgical teams, including the present authors, to reduce operative morbidity, especially facial paralysis. With such tumours the authors have found that the facial nerve can be more readily identified and preserved from a lateral approach than from a suboccipital approach. ${ }^{4}$ With meningiomas, preoperative hearing is not usually irreparably damaged, and therefore, in most instances, a suboccipital approach is preferred to allow preservation of hearing. In our view, with the suboccipital approach, it is easier to separate meningiomas from the adjacent blood vessels and thoroughly remove them from their dural attachment which may be very extensive. However, meningiomas with major superior or anterior extensions should be approached from a lateral approach, and as shown in Table 7, nine tumours in this series were removed by a translabyrinthine approach, mainly the expanded translabyrinthine approach.

The third reason for accurate preoperative diagnosis is to alert the surgeon about the location of the seventh and eighth cranial nerves in relationship to the tumour. Acoustic neuromas almost always displace the seventh and eighth cranial nerves anteriorly, or anteromedially, whereas the meningiomas almost always displace the nerves posteriorly or posterolaterally. With meningiomas, the nerves can be easily injured during tumour removal if the surgeon mistakes the tumour for an acoustic neuroma. Searching the surface of the tumour with an electrode is invaluable for detecting the facial nerve. ${ }^{2}$

\section{Conclusions}

Next to acoustic neuromas, meningiomas are the most common type of primary tumour in the posterior fossa. CPA meningiomas growing from either the petrous bone or from the tentorium may mimic acoustic neuromas in both clinical and radiological features. However, there are important distinguishing features. The meningiomas are much more common in females and tend to be larger tumours. They more commonly produce trigeminal symptoms with less involvement of hearing. On CT the meningiomas are more sessile, with a broader base along the tentorium or petrous ridge, and without expansion of the internal auditory canal. The MR is the best test for distinguishing the two types. In most instances, posterior fossa meningiomas can be totally excised with low rates of morbidity, and in modern series, the mortality rate has been very low.

\section{REFERENCES}

1. Castellano F, Ruggiero G. Meningiomas of the posterior fossa. Acta Radiol Suppl 1953; 104: 1-164.

2. Tator $\mathrm{CH}$, Linden RD, Duncan EG. Management of acoustic neuromas in 212 patients. In: Fraysse B and Lazorthes Y Eds. Neurinomes de L'Acoustique; Tome 2 Paris, Editons Medicales Pierre Fabre, 1988.

3. Tator $\mathrm{CH}$. Acoustic neuromas: Management of 204 cases. Can J Neurol Sci 1985; 12: 353-357.

4. Tator $\mathrm{CH}$, Nedzelski JM. Facial nerve preservation in patients with large acoustic neuromas treated by a combined middle fossa transtentorial translabyrinthine approach. J Neurosurg 1982; 57: $1-7$. 
5. Tator $\mathrm{CH}$, Nedzelski JM. Preservation of hearing in patients undergoing excision of acoustic neuromas and other cerebellopontine angle tumours. J Neurosurg 1985; 63: 168-174.

6. Miller R. Meningiomas of the posterior fossa. In: Bucheit W, Truex RC Jr, Eds. Surgery of the Posterior Fossa. New York: Raven Press 1979: 99-110.

7. Sekhar LN, Jannetta PJ. Cerebellopontine angle meningiomas. Microsurgical excision and follow-up results. J Neurosurg 1984; 60: 500-505.

8. Cushing HW, Eisenhardt L. Meningiomas: their classification, regional behaviour, life history, and surgical results. Springfield, III: Charles C Thomas 1938; 3-387.

9. Petit-Dutaillis D, Daum S. Les meningiomes de la fosse posterieure: Rev Neurol 1949; 81: 557.572.

10. Russell JR, Bucy PC. Meningiomas of the posterior fossa. Surg Gynecol Obstet 1953; 96: 183-192.
11. Markham JW, Fager CA, Horrax G, et al. Meningiomas of the posterior fossa. Their diagnosis, clinical features, and surgical treatment. Arch Neurol Psychiatry 1955; 74: 1263-170.

12. Hoffmann GR, De Busscher J, De Haene A. Le meningiome de l'angle pontocerebelleux, Neurochirurgia (Stuttg.) 1957; 3: 123 137.

13. Scott $M$. The surgical management of the meningiomas of the posterior fossa. Surg Gynecol Obstet 1972; 135: 545-550.

14. Yasargil MG, Mortara RW, Curcic M. Meningiomas of the basal posterior cranial fossa. $I n$ : Krayenbuhl H, Ed. Advances and Technical Standards in Neurosurgery. Wien, Springer-Verlag 1980: 3-115.

15. Theron $J$ and Lasjaunias P. Participation of the external and internal carotid arteries in the blood supply of acoustic neuromas. Radiology 1976; 118: 83-88. 